\title{
Treatment options in early triple-negative breast cancer
}

\section{Update from the San Antonio Breast Cancer Symposium 2019}

\author{
Christoph Suppan · Marija Balic
}

Received: 8 April 2020 / Accepted: 14 April 2020 / Published online: 7 May 2020

(C) The Author(s) 2020

\begin{abstract}
Summary With a main focus on the early stage triple-negative breast cancer (TNBC), new data on immunotherapy in combination with chemotherapy, the role of capecitabine, the potential of circulating tumor DNA as a predictive tool in the postneoadjuvant setting and new treatment approaches were presented and discussed at the San Antonio Breast Cancer Symposium (SABCS) 2019.
\end{abstract}

Keywords Immunotherapy - Chemotherapy · pCR . PD-Ll inhibition $\cdot$ ctDNA

Triple-negative breast cancer (TNBC) remains the most aggressive subtype of breast cancer with a high risk of recurrence in early stages and an unfavorable prognosis [1]. In light of preliminary results obtained with immune checkpoint inhibitors and the benefit in overall survival in PD-L1-positive patients with metastatic TNBC undergoing nab-paclitaxel and atezolizumab [2], these agents are already under evaluation in combination with chemotherapy in the neoadjuvant setting. At the San Antonio Breast Cancer Symposium in 2019, two phase III trials in early stage TNBC were presented, investigating the potential role of PD-1/PD-L1 inhibitors added to chemotherapy to enhance the rate of pathologic complete response (pCR).

KEYNOTE-522 enrolled 1174 patients with stage II or III TNBC treated with neoadjuvant and adjuvant systemic therapy. Preoperatively, patients were randomized to receive pembrolizumab at a dose of $200 \mathrm{mg}$ every 3 weeks versus placebo in combina-

\section{Suppan $(\bowtie) \cdot$ M. Balic}

Division of Oncology, Department of Internal Medicine,

Medical University of Graz, Auenbruggerplatz, 15, 8036 Graz, Austria

christoph.suppan@klinikum-graz.at tion with chemotherapy. Chemotherapy consisted of 12 weeks of carboplatin plus paclitaxel, followed by epirubicin or doxorubicin with cyclophosphamide every 3 weeks for four cycles. After surgery, patients received another nine cycles of pembrolizumab or placebo. The co-primary endpoints were pCR and event-free survival (EFS). About $75 \%$ of patients had $\mathrm{T} 1$ or T2 tumors, $51 \%$ were node-positive and more than $80 \%$ were PD-L1-positive based on a combined positive score (CPS) $\geq 1$. The pCR rate in the pembrolizumab arm was $64.8 \%$ versus $51.2 \%$ in the placebo arm, resulting in an absolute difference of 13.6\% ( $p=0.00055$ ). The benefit of pembrolizumab was independent of the PD-Ll status. At a median follow-up of 15.5 months, EFS in the pembrolizumab arm was $91.3 \%$ versus $85.3 \%$ in the placebo arm. The greatest benefit of added pembrolizumab was seen in stage IIIB patients with a pCR of $48.6 \%$ versus $23.1 \%$ in the placebo arm. Patients with node-positive disease also had a greater benefit with a pCR rate of $64.8 \%$ when receiving pembrolizumab versus $44.1 \%$ treated with placebo. The immune-mediated adverse event rates were consistent with the known profiles of each regimen and no new safety signals could be observed. The EFS results are immature due to a median follow-up of 15.5 months and have to be confirmed in a longer follow-up along with the long-term safety profile [3].

The NeoTRIP study was the second trial presented in a similar patient population, investigating the role of adding atezolizumab to chemotherapy. Here, 280 patients with early TNBC were randomized to receive either atezolizumab at a dose of $1200 \mathrm{mg}$ every 3 weeks or placebo in combination with carboplatin and nab-paclitaxel for eight cycles. All patients underwent surgery and then received four cycles of anthracycline-based chemotherapy as per investigators' choice. About half of the patients had locally 
advanced disease, $56 \%$ were $\mathrm{PD}-\mathrm{Ll}$ positive and $13 \%$ had node-negative disease. The primary endpoint was EFS at 5 years following randomization of the last patient, and the secondary endpoint was pCR. The pCR rate in the atezolizumab arm was $43.5 \%$ versus $40.8 \%$ in the placebo arm, which was not statistically significant with a $p$-value of 0.66 . In multivariate analysis, only PD-L1 positivity was significantly associated with pCR $(p<0.0001)$. The rate of clinical overall response was $76.1 \%$ under added atezolizumab versus $68.3 \%$ in the placebo arm. Treatment-related adverse events were similar in both regimens except for a significantly higher overall incidence of serious adverse events and liver transaminase abnormalities with atezolizumab [4].

There are several reasons responsible for the different results. First, the type of chemotherapy backbone and the treatment duration were different. Second, the difference may be associated with different targets of the drugs, the one inhibiting PD-1 and the other PD-L1. And third, the data are at an early time point, lacking long-term effects. However, the results of the KEYNOTE-522 in particular are promising, although there are still unanswered questions. At this time point, it is too early to incorporate this treatment as a new standard of care.

Another neoadjuvant study presented at the meeting was a trial addressing the role of platin therapy in germline BRCA-mutated patients in the phase II INFORM trial. Here, 117 patients with a germline BRCA1 or BRCA2 mutation and HER2-negative tumors were randomly assigned to four cycles of cisplatin at a dose of $75 \mathrm{mg} / \mathrm{m}^{2}$ every 3 weeks or doxorubicin and cyclophosphamide (AC) for four cycles every 2-3 weeks. pCR - the primary endpoint of the study - in patients with cisplatin was $18 \%$ versus $26 \%$ in the $\mathrm{AC}$ arm. In triple-negative breast cancer patients, pCR was $22 \%$ with cisplatin versus $28 \%$ with AC. This difference was not statistically significant and both results were lower than previously reported in germline BRCA carriers [5]. However, this trial did not strengthen the role of platins in BRCA-positive patients.

A new approach in the treatment of metastatic TNBC patients was presented in the phase II KEYNOTE-890 trial. In this single-arm study, 25 patients with at least one line of prior systemic chemotherapy with or without immunotherapy were included and treated with intratumoral tavokinogene telseplasmid, electroporation and pembrolizumab. The injection of tavokinogene telseplasmid into the tumor followed by electroporation in the same region leads to destabilization of the tumor membrane through electrical pulses and an uptake of interleukin-12 coded DNA, thereby stimulating a proinflammatory immune response. The combination with the PD-1 inhibitor pembrolizumab may convert tumors that are poorly immunogenic into immunoresponsive tumors. Indeed, partial responses were seen in $28.6 \%$ of patients, whereas three of four were negative for PD-L1 expression [6]. These results represent a promising approach for future studies.

The role of capecitabine as a part of systemic therapies in early breast cancer patients was discussed after the presentation of a meta-analysis of over 15,000 patients. This meta-analysis included 12 randomized controlled trials: in five of them, patients received capecitabine instead of other therapies, whereas the other 7 studies investigated the role of the addition of capecitabine to systemic therapy. About $75 \%$ of patients had node-positive disease, $68 \%$ were hormone receptor-positive, $56 \%$ presented with stage II disease and $15 \%$ were HER2-positive. In the overall study population, about $80 \%$ were treated in the adjuvant setting and $20 \%$ neoadjuvantly. There was no significant effect of capecitabine on disease-free survival (DFS), but patients who received capecitabine in addition to other systemic therapies had a significant benefit $(\mathrm{HR}=0.88)$. Moreover, there was a small benefit of capecitabine in overall survival $(\mathrm{HR}=0.982, p=0.005$ ) and when it was used in addition to other systemic therapies $(\mathrm{HR}=0.892, p=0.001)$. In TNBC patients, the addition of capecitabine improved DFS by $18 \%$ and OS by $22 \%(P=0.04)$. However, no benefit was seen when capecitabine was used instead of another systemic therapy in TNBC patients. It is important to notice, that only the Create-X trial had positive results for DFS, and together with the USON 01062 trial, positive results for overall survival, as well. Altogether, the addition of capecitabine to standard chemotherapy can be recommended to TNBC patients with a high risk of recurrence [7].

The phase II BRE12-158 trial attempted to answer the question as to which of the early stage TNBC patients after surgery and neoadjuvant chemotherapy would need further therapy or have a high risk of recurrence. It investigated the potential of using circulating tumor DNA (ctDNA) and circulating tumor cells (CTCs) to detect minimal residual disease. ctDNA sequencing was performed by the FoundationOne Liquid Test, which tests 70 commonly mutated oncogenes; CTCs were isolated from peripheral blood using EpCAM-based positive selection microfluidic device. CTC-positivity was defined as $\geq 1$ CTC detected. The analysis was performed at a median clinical follow-up of 17.2 months.

The detection of ctDNA was significantly associated with a lower distant disease-free survival (median 32.5 months versus not reached, $p=0.003$ ). The CTC-positivity demonstrated a trend for association with inferior outcomes, but did not reach statistical significance. The absence of both CTCs and ctDNA had a superior ability for predicting no relapse. The authors concluded that especially the detection of ctDNA in TNBC patients after neoadjuvant therapy and surgery is an independent predictor of disease recurrence and an important stratification factor for upcoming postneoadjuvant trials [8]. 


\section{Take home message}

The majority of patients with TNBC have a poor prognosis and often develop resistance to chemotherapy in a fast way. In light of the presented data on immunotherapy, there is new hope for patients with early stage disease.

Funding Open access funding provided by Medical University of Graz.

Conflict of interest C. Suppan and M. Balic declare that they have no competing interests.

Open Access This article is licensed under a Creative Commons Attribution 4.0 International License, which permits use, sharing, adaptation, distribution and reproduction in any medium or format, as long as you give appropriate credit to the original author(s) and the source, provide a link to the Creative Commons licence, and indicate if changes were made. The images or other third party material in this article are included in the article's Creative Commons licence, unless indicated otherwise in a credit line to the material. If material is not included in the article's Creative Commons licence and your intended use is not permitted by statutory regulation or exceeds the permitted use, you will need to obtain permission directly from the copyright holder. To view a copy of this licence, visit http://creativecommons.org/licenses/by/4.0/.

\section{References}

1. Waks AG, Winer EP. Breast cancer treatment-a review. JAMA. 2019;321(3):288-300.

2. Schmid P, Adams S, Rugo HS, et al. Atezolizumab and nabpaclitaxel in advanced triple-negative breast cancer. NEngl JMed. 2018;379(22):2108-21.

3. Schmid P, Park YH, Ferreira M, et al. KEYNOTE-522: phase 3 study of neoadjuvant pembrolizumab plus chemotherapy versus placebo plus chemotherapy, followed by adjuvant pembrolizumab versus placebo for early triple-negative breast cancer. San Antonio: Breast Cancer Symposium; 2019. Abstract GS3-03.
4. Gianni L, Huang C, Egle D, et al. Pathologic complete response to neoadjuvant treatment with or without atezolizumab in triple-negative, early high-risk and locally advanced breast cancer. NeoTRIPaPDL1 Michelangelo randomized study. San Antonio: Breast Cancer Symposium;2019. AbstractGS3-04.

5. Tung N, Arun B, Hofstatter E, et al. Cisplatin versus doxorubicin/cyclophosphamideasneoadjuvant treatment in germline BRCA mutation carriers (BRCA carriers) with HER2-negative breast cancer: results from the INFORM trial (TBCRC 031). San Antonio: Breast Cancer Symposium; 2019. AbstractGS6-03.

6. Telli ML, Wapnir I, Devitt B, et al. Phase II, open-label study of intratumoral tavokinogene telseplasmid (tavo) plus electroporation in combination with intravenous pembrolizumab therapy in patients with inoperable locally advanced or metastatic triple-negative breast cancer. San Antonio: Breast Cancer Symposium; 2019. Abstract P309-04.

7. van Mackelenbergh M, Seither F, Möbus V, et al. Effects of capecitabine as part of neo-/adjuvant chemotherapy: a meta-analysis of individual patient data from 12 randomized trials including 15,457 patients. San Antonio: Breast Cancer Symposium; 2019. Abstract GS1-07.

8. Radovich M, Jiang G, Chitambar C, et al. Detection of circulating tumor DNA (ctDNA) after neoadjuvant chemotherapy is significantly associated with disease recurrence in early-stage triple-negative breast cancer (TNBC): preplanned correlative results from clinical trial BRE12-158. San Antonio: Breast Cancer Symposium; 2019. Abstract GS5-02.

Publisher's Note Springer Nature remains neutral with regard to jurisdictional claims in published maps and institutional affiliations.

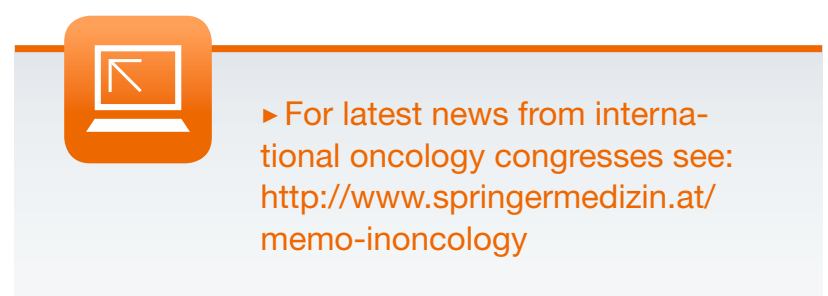

